\title{
The Effect of Elution Volume for Immunoprecipitation on $\mathbf{m}^{6} \mathrm{~A}-\mathrm{Seq}$ Analysis
}

\author{
Yuhui Xu' ${ }^{1}$, Lize Shen ${ }^{2}$, Guolin $\mathrm{Li}^{1 *}$ \\ ${ }^{1}$ College of Life Sciences, Hunan Normal University, Changsha 410081, Hunan Province, China \\ ${ }^{2}$ R\&D Department, LC-Bio Technology Co., Ltd, Hangzhou 310018, Zhejiang Province, China \\ *Corresponding author: Guolin Li, hnsdlgl@hunnu.edu.cn
}

\begin{abstract}
Objective: To develop a cost-effective method to reduce the time consumption of elution in immunoprecipitation. Method: Two volumes ( $125 \mu \mathrm{L}$ for Group C and $100 \mu \mathrm{L}$ for Group T) of elution buffer were used to explore whether smaller volume could save testing time. Result: Time consumption of elution in Group T was significantly shorter than that in Group $\mathrm{C}$, while the efficiency of eluted $\mathrm{m}^{6} \mathrm{~A}$-containing fragments and the performance of $\mathrm{m}^{6} \mathrm{~A}$-Seq as indicated by $\mathrm{m}^{6} \mathrm{~A}$ peak distributions showed no difference between the two groups. Conclusion: A smaller volume of elution buffer was an economical way to reduce time consumption in immunoprecipitation.
\end{abstract}

Keywords: $\mathrm{m}^{6} \mathrm{~A}-\mathrm{Seq}$; Immunoprecipitation; Elution buffer

Publication date: July 2021; Online publication: July 30, 2021

\section{Introduction}

Many studies have shown that N6-methyladenosine ( $\left.m^{6} \mathrm{~A}\right)$ RNA modification plays a key role in biological process, especially in the initiation and progression of different types of human cancers ${ }^{[1]}$. In 2012 , the $\mathrm{m}^{6} \mathrm{~A}$ landscape in humans and mice at whole transcriptional level was revealed by an immunoprecipitationbased next generation sequencing method called $\mathrm{m}^{6} \mathrm{~A}-\mathrm{Seq}$ (also known as MeRIP-Seq) ${ }^{[2-3]}$. However, $\mathrm{m}^{6} \mathrm{~A}-$ Seq were still limited by time consumption of immunoprecipitation, higher amount of total RNA input for immunoprecipitation, bacterial contamination, etc. In this study, we reduced the elution volume of buffer in order to develop a more cost-effective method for immunoprecipitation and reduce time consumption during the whole experiment cycle.

\section{Materials and methods}

\subsection{Cell line}

The Mouse Renal Carcinoma cells (RenCa) were cultured in DMEM medium (Invitrogen) containing 10\% FBS, 2 mM L-Glutamine and 1\% penicillin-streptomycin. RenCa cell line was purchased from Allcells Biotechnology (Shanghai, China) and were routinely tested for mycoplasma contamination.

\subsection{Equipment}

\begin{tabular}{lll}
\hline Equipment/resource & Source & Identifier \\
\hline Water bath & Shanghai Boxun & DK-8D \\
Room temperature centrifuge & Eppendorf & 5415D \\
Refrigerated centrifuge & Eppendorf & $5418 \mathrm{R}$ \\
\hline
\end{tabular}

(Continued next page) 
(Continued)

\begin{tabular}{lll}
\hline Equipment/resource & Source & Identifier \\
\hline Vertical mixer & Ningbo Xinzhi & HS-3 \\
NanoDrop ND-1000 & NanoDrop & ND-1000 \\
Bioanalyzer 2100 & Agilent & G2939BA \\
Pipettor & Eppendorf & L17490H \\
\hline
\end{tabular}

\subsection{Reagent and buffer}

\begin{tabular}{llll}
\hline Reagent/resource & Source & Identifier & Storage $\left({ }^{\circ} \mathbf{C}\right)$ \\
\hline TRIzol & Invitrogen & 15596026 & $2-8^{\circ} \mathrm{C}$ \\
Dynabeads ${ }^{\circ}$ Oligo (dT) & Thermo Fisher & $25-61005$ & $2-8^{\circ} \mathrm{C}$ \\
$m^{6} \mathrm{~A}$ antibody & Synaptic Systems (SYSY) & 202003 & $-20^{\circ} \mathrm{C}$ \\
Magnesium RNA Fragmentation Module & New England Biolabs, Inc & E6150S & $-20^{\circ} \mathrm{C}$ \\
Dynabeads Antibody Coupling Kit & Thermo Fisher & $14311 \mathrm{D}$ & $2-8^{\circ} \mathrm{C}$ \\
SuperScript ${ }^{\mathrm{TM}}$ II Reverse Transcriptase & Invitrogen & 1896649 & $-20^{\circ} \mathrm{C}$ \\
E. coli DNA polymerase I & New England Biolabs, Inc & $\mathrm{m} 0209$ & $-20^{\circ} \mathrm{C}$ \\
RNase H & New England Biolabs, Inc & $\mathrm{m} 0297$ & $-20^{\circ} \mathrm{C}$ \\
dUTP Solution & Thermo Fisher & $\mathrm{R} 0133$ & $-20^{\circ} \mathrm{C}$ \\
UDG enzyme & New England Biolabs, Inc & $\mathrm{m} 0280$ & $-20^{\circ} \mathrm{C}$ \\
Truseq stranded mRNA library prep kit & Illumina & 15031047 & $-20^{\circ} \mathrm{C}$ \\
\hline
\end{tabular}

\begin{tabular}{ll}
\hline Buffer & Components \\
\hline Fragment buffer & $10 \mathrm{mM} \mathrm{ZnCl} 2,10 \mathrm{mM}$ Tris- $\mathrm{HCl}(\mathrm{pH} 7.0)$ \\
Fragment stop buffer & $0.5 \mathrm{M}$ EDTA \\
$\mathrm{m}^{6} \mathrm{~A}$ binding buffer & $50 \mathrm{mM}$ Tris- $\mathrm{HCl}(\mathrm{pH} 7.4), 150 \mathrm{mM} \mathrm{NaCl}, 1 \% \mathrm{NP}-40,2 \mathrm{mM}$ EDTA \\
Low salt buffer & $0.2 \times \mathrm{SSPE}, 0.001 \mathrm{M}$ EDTA, 0.05\% Tween-20 \\
High salt buffer & $0.2 \times \mathrm{SSPE}, 0.001 \mathrm{M}$ EDTA, 0.05\%, Tween-20, 137.5 mM NaCl \\
TET buffer & $10 \mathrm{mM}$ Tris- $\mathrm{HCl}(\mathrm{pH} 8.0), 1 \mathrm{mM}$ EDTA $(\mathrm{pH} 8.0), 0.05 \%$ Tween-20 \\
Elution buffer & $0.02 \mathrm{M}$ DTT, $0.150 \mathrm{M} \mathrm{NaCl}, 0.05 \mathrm{M}$ Tris-HCl $(\mathrm{pH} 7.5), 0.001 \mathrm{M}$ EDTA, 0.10\% SDS \\
\hline
\end{tabular}

\subsection{RNA extraction and mRNA enrichment}

Total RNA of cell line was isolated and purified using TRIzol reagent (Invitrogen, CA, USA) following the manufacturer's procedure. More than $300 \mu \mathrm{g}$ total RNA was yielded by RenCa cell $\left(3 \times 10^{8}\right)$ in each sample. The RNA amount and purity were quantified using NanoDrop ND-1000 (NanoDrop, Wilmington, DE, USA). The RNA integrity was assessed by Bioanalyzer 2100 (Agilent, CA, USA) with RNA integrity number $(\mathrm{RIN})>7.0$ and confirmed by electrophoresis with denaturing agarose gel. Poly(A) RNA was purified from $300 \mu \mathrm{g}$ total RNA using Dynabeads Oligo (dT) 25-61005 (Thermo Fisher, CA, USA) in four rounds of purification. Then, the poly(A) RNA was fragmented into small pieces using Magnesium RNA Fragmentation Module (NEB, cat.e6150, USA) under $86^{\circ} \mathrm{C}$ for $7 \mathrm{~min}$. Amount of fragmented Poly(A) enriched RNA was $3458.4 \mathrm{ng}$ and we equally separated into six tubes named with C1, C2, C3, T1, T2, and T3.

\subsection{Immunoprecipitation}

$500 \mathrm{ng}$ of poly(A) RNA was used for $\mathrm{m}^{6} \mathrm{~A}$-Seq in each biological replicate. $140 \mu \mathrm{L}$ of pre-equilibrated $\mathrm{m}^{6} \mathrm{~A}$-Dynabeads was added to the $360 \mu \mathrm{L}$ of fragmented RNA to a final volume of $500 \mu \mathrm{L}$. The fragmented 
RNA was allowed to bind the $\mathrm{m}^{6} \mathrm{~A}-\mathrm{Dy}$ nabeads at room temperature for $1 \mathrm{~h}$. The tubes containing the samples were placed on a magnet allowing the bead complexes to cluster until the solution became clear. The liquid phase or supernatant in this $500 \mu \mathrm{L}$ was discarded as this fraction represented the $\mathrm{m}^{6} \mathrm{~A}$ negative fragments, which was not captured by the antibody. The $\mathrm{m}^{6} \mathrm{~A}$-Dynabeads-RNA complexes was resuspended and incubated in $500 \mu \mathrm{L}$ of $\mathrm{m}^{6} \mathrm{~A}$ Binding Buffer for $3 \mathrm{~min}$ at room temperature, and clear supernatant was removed after placing the beads in the magnet. Finally, high salt buffer, low salt buffer and TET buffer were added subsequently.

For immunoprecipitation, each biological replicate in Group T (T1, T2, T3) was treated with $100 \mu \mathrm{L}$ elution buffer, while each biological replicate in Group $\mathrm{C}(\mathrm{C} 1, \mathrm{C} 2, \mathrm{C} 3)$ was treated with $125 \mu \mathrm{L}$ elution buffer.

\subsection{Library construction and sequencing}

The eluted $\mathrm{m}^{6} \mathrm{~A}$-containing fragments (IP) and untreated input control fragments were reverse-transcribed to create cDNA libraries by SuperScript ${ }^{\mathrm{TM}}$ II Reverse Transcriptase (Invitrogen, cat. 1896649, USA), which were then used to synthesize U-labeled second-stranded DNAs with E. coli DNA polymerase I (NEB, cat.m0209, USA), RNase H (NEB, cat.m0297, USA) and dUTP Solution (Thermo Fisher, cat.R0133, USA). An A-base was then added to the blunt ends of each strand, preparing them for ligation to the indexed adapters. Each adapter contained a T-base overhang for ligating the adapter to the A-tailed fragmented DNA. Single- or dual-index adapters were ligated to the fragments, and size selection was performed using AMPureXP beads. After the heat-labile UDG enzyme (NEB, cat.m0280, USA) treatment of the U-labeled second-stranded DNAs, the ligated products were amplified with PCR by the following conditions: initial denaturation at $95^{\circ} \mathrm{C}$ for $3 \mathrm{~min}, 8$ cycles of denaturation at $98^{\circ} \mathrm{C}$ for $15 \mathrm{sec}$, annealing at $60^{\circ} \mathrm{C}$ for $15 \mathrm{sec}$, extension at $72^{\circ} \mathrm{C}$ for $30 \mathrm{sec}$, and final extension at $72^{\circ} \mathrm{C}$ for $5 \mathrm{~min}$. The average insert size for the final cDNA library was $300 \pm 50 \mathrm{bp}$. At last, $2 \times 150 \mathrm{bp}$ paired-end sequencing (PE150) was performed on an Illumina Novaseq ${ }^{\mathrm{TM}} 6000$ (LC-Bio Technology Co., Ltd., Hangzhou, China) following the manufacturer's recommended protocol.

\subsection{Bioinformatic analysis of $\mathrm{m}^{\mathbf{6}} \mathrm{A}-\mathrm{Seq}$}

The fastp software (https://github.com/OpenGene/fastp) were used to remove the reads containing adaptor contamination, low quality bases and undetermined bases with default parameters. Then sequence quality of IP and Input samples were also verified using fastp. We used HISAT2 (http://daehwankimlab.github.io/hisat2) to map reads to the reference genome of Mus musculus (Ensembl Version: v96). The mapped reads of IP and input libraries were loaded into $\mathrm{R}$ package exomePeak (https://bioconductor.org/packages/exomePeak), which could identify $\mathrm{m}^{6} \mathrm{~A}$ peaks with bed or bigwig format that could be adapted for visualization on the IGV software (http://www.igv.org). Called peaks were annotated by intersection with gene architecture using $\mathrm{R}$ package ChIPseeker (https://bioconductor.org/packages/ChIPseeker).

\subsection{Statistical methods}

Student's $t$ test (t.test function) was utilized for data processing. The $P$ value less than $0.01(P<0.01)$ was considered statistically significant.

\section{Result}

\subsection{Less elution volume saves elution time}

The IP efficiency of $\mathrm{m}^{6} \mathrm{~A}$ modified RNA eluted by Group $\mathrm{T}(6.267 \% \pm 0.64)$ and Group C $(5.923 \% \pm 1.07)$ was not significant difference $(P>0.1)$ (Figure 1A). However, the time consumption of elution in Group 
T $(109.67 \pm 3.05 \mathrm{~min})$ was significantly shorter $(P<0.001)$ than that in Group $C(194 \pm 8.89$ min) (Figure 1B).
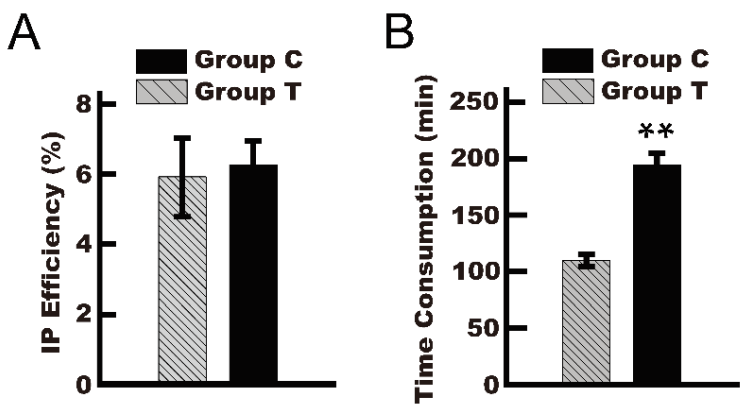

Figure 1. Less elution volume saves elution time. The immunoprecipitation (IP) efficiency (A) and time consumption (B) of elution in Group $\mathrm{C}(\mathrm{n}=3)$ and Group $\mathrm{T}(\mathrm{n}=3)$. Data were presented as mean \pm SEM, and $* *$ represented $P<0.001$ based on student's t test.

\subsection{Less elution volume does not affect the distribution of $\mathbf{m}^{6} \mathrm{~A}$ peaks in $\mathrm{m}^{6} \mathrm{~A}-\mathrm{Seq}$}

We next characterized the distribution of $\mathrm{m}^{6} \mathrm{~A}$ peaks in the whole transcriptome of all samples. The metagenomic profiles of $\mathrm{m}^{6} \mathrm{~A}$ peaks in both Group $\mathrm{C}$ and Group $\mathrm{T}$ indicated that $\mathrm{m}^{6} \mathrm{~A}$ modifications were highly enriched around the 5' stop codon untranslated region (UTR) and 3' UTR (Figure 2A).

To confirm the distribution of $\mathrm{m}^{6} \mathrm{~A}$ within the transcript, we divided the transcript into four nonoverlapping segments: 5' UTR, 3' UTR, the first Exon and other Exons. Each $\mathrm{m}^{6} \mathrm{~A}$ peak was assigned into one of the four transcript segments. The 3' UTR appeared to be greatly enriched in $\mathrm{m}^{6} \mathrm{~A}$ peaks, and $49.82 \%$ to $50.13 \%$ of the peaks from the two groups fell into this segment (Figure 2B).

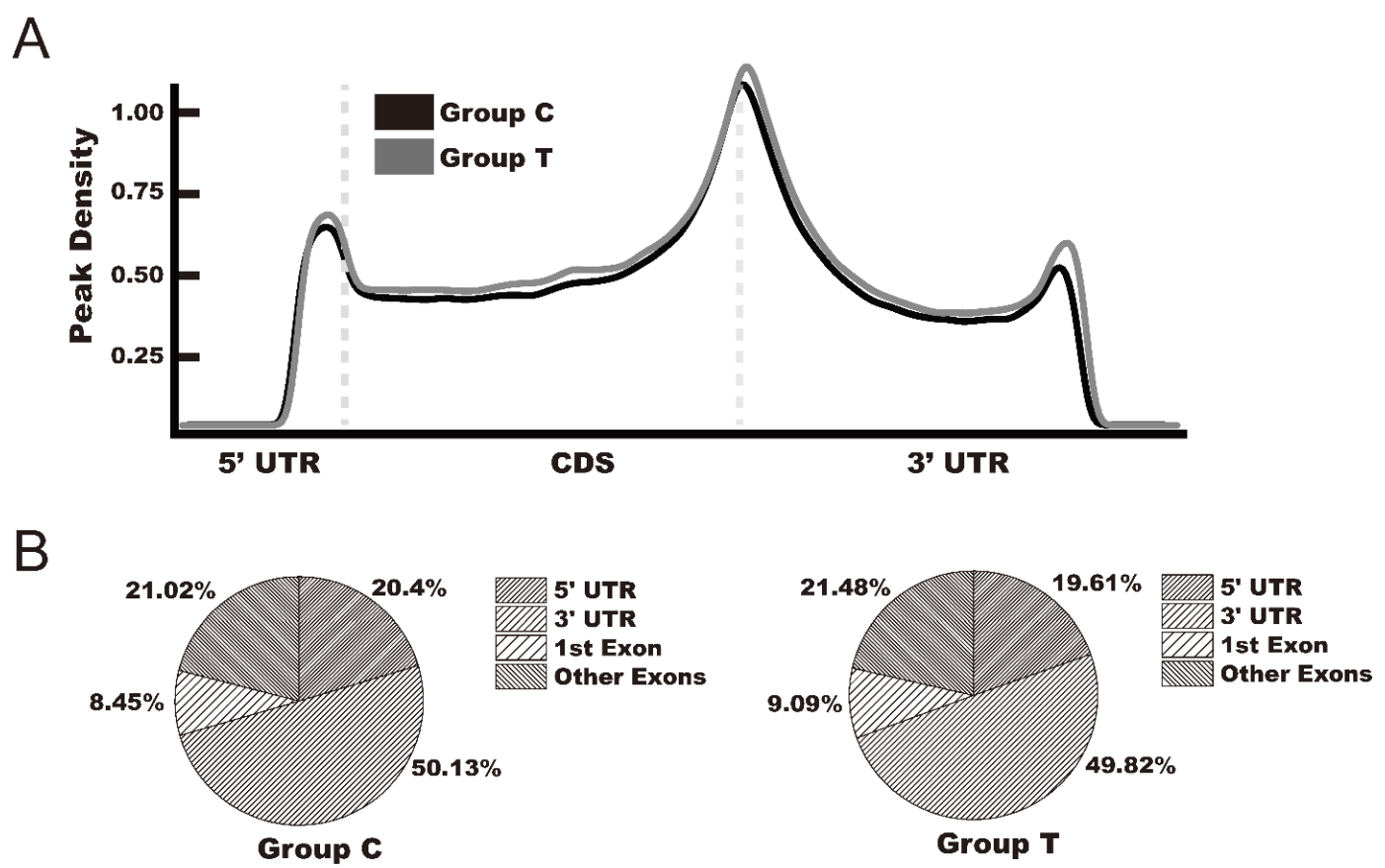

Figure 2. Lower elution volume does not affect the distribution of $\mathrm{m}^{6} \mathrm{~A}$ peaks in $\mathrm{m}^{6} \mathrm{~A}$-Seq. Epi-Transcriptome analysis of total $\mathrm{m}^{6} \mathrm{~A}$ peaks in Group $\mathrm{C}(\mathrm{n}=3)$ and Group $\mathrm{T}(\mathrm{n}=3)$. (A) Metagenomic profiles of $\mathrm{m}^{6} \mathrm{~A}$ peak summit distributions along the transcripts composed of 5' UTR, CDS, and 3' UTR. (B) Pie charts depicting the distribution of $\mathrm{m}^{6} \mathrm{~A}$ peak within four nonoverlapping gene regions (5' UTR, 3' UTR, first exon, other exons). 
These results were consistent with the $\mathrm{m}^{6} \mathrm{~A}$ distribution in previous studies of $\mathrm{m}^{6} \mathrm{~A}$ modification landscape in human and mouse ${ }^{[2-3]}$, suggesting that the modified IP assay and library construction were successful.

\section{Discussion}

A classic protocol for $\mathrm{m}^{6} \mathrm{~A}-\mathrm{Seq}{ }^{[2-3]}$ included RNA extraction, poly(A) RNA enrichment, RNA binding to $\mathrm{m}^{6} \mathrm{~A}$-Dynabeads, washing of $\mathrm{m}^{6} \mathrm{~A}$-Dynabeads, elution of $\mathrm{m}^{6} \mathrm{~A}$-positive RNA, library construction, and Illumina sequencing. These steps may account for more than 36 hours, of which more than 2 hours will be spent on the elution step. The content of eluted $\mathrm{m}^{6} \mathrm{~A}$-containing fragments for library construction is no more than $50 \mathrm{ng}$. On the basis of canonical method for $\mathrm{m}^{6} \mathrm{~A}$ immunoprecipitation, we reduced elution volume buffer from $125 \mu \mathrm{L}$ to $100 \mu \mathrm{L}$. Lower volume of elution buffer could save almost 100 min than higher volume, but the performance of $\mathrm{m}^{6} \mathrm{~A}-\mathrm{Seq}$ proved that $\mathrm{m}^{6} \mathrm{~A}$ peaks distributed on gene regions had no difference between the two conditions. In conclusion, $100 \mu \mathrm{L}$ elution buffer could save almost half of the working time in IP elution than the step using $125 \mu \mathrm{L}$ while maintaining similar performance in $\mathrm{m}^{6} \mathrm{~A}$-Seq.

\section{Acknowledgments}

We thank Mr Jinlong Kuang in LC-Bio Technology for his help with the sample collection process.

\section{Disclosure statement}

The authors declare no conflict of interest.

\section{Author contributions}

Yuhui $\mathrm{Xu}$, Lize Shen, Guolin Li designed the experiments, performed all the experiments, and wrote the manuscript. Lize Shen designed and performed the bioinformatics analysis. All the authors commented and approved the paper.

\section{References}

[1] Jiang XL, Liu BY, Nie Z, et al., 2021, The Role of $\mathrm{m}^{6} \mathrm{~A}$ Modification in the Biological Functions and Diseases. Signal Transduction and Targeted Therapy, 6(1): 74.

[2] Meyer KD, Saletore Y, Zumbo P, et al., 2012, Comprehensive Analysis of MRNA Methylation Reveals Enrichment in 3' UTRS and Near Stop Codons. Cell, 149(7): 1635-1646.

[3] Dominissini D, Moshkovitz S, Schwartz, et al., 2012, Topology of the Human and Mouse $m^{6}$ a RNA Methylomes Revealed by m6-Seq. Nature, 485(7397): 201-206. 